\title{
Biomimetic Nanopillar-Based Biosensor for Label-Free Detection of Influenza A Virus
}

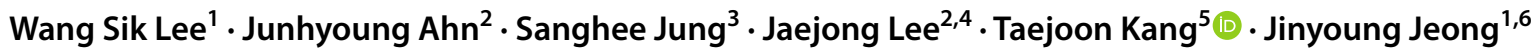

Received: 13 January 2021 / Revised: 12 May 2021 / Accepted: 17 May 2021 / Published online: 8 June 2021

(c) The Author(s), under exclusive licence to The Korean BioChip Society 2021

\begin{abstract}
Since the first emergence of influenza viruses, they have caused the flu seasonally worldwide. Precise detection of influenza viruses is required to prevent the spreading of the disease. Herein, we developed an optical biosensor using peptide-immobilized nanopillar structures for the label-free detection of influenza viruses. The spin-on-glass nanopillar structures were fabricated by nanoimprint lithography. A sialic acid-mimic peptide, which can specifically bind to hemagglutinin on the surface of the influenza virus, was immobilized onto the nanopillars via polymerized dopamine. The constructed nanopillar sensor enabled us to detect influenza A viruses in the range of $10^{3}-10^{5}$ plaque-forming units through simple measurements of reflectance. Our findings suggest that biomimetic modification of nanopillar structures can be an alternative method for the immunodiagnosis of influenza viruses.
\end{abstract}

Keywords Nanopillar $\cdot$ Influenza virus $\cdot$ Peptide $\cdot$ Polydopamine $\cdot$ Reflectance

\section{Introduction}

Jaejong Lee

jjlee@kimm.re.kr

$\triangle$ Taejoon Kang

kangtaejoon@kribb.re.kr

Jinyoung Jeong

jyjeong@kribb.re.kr

1 Environmental Disease Research Center, Korea Research Institute of Bioscience and Biotechnology (KRIBB), 125

Gwahak-ro, Yuseong-Gu, Daejeon 34141, Republic of Korea

2 Nano-Convergence Mechanical Systems Research Division, Korea Institute of Machinery and Materials, 156 Gajeongbuk-ro, Yuseong-gu, Daejeon 34103, Republic of Korea

3 Office of Nano-Convergence Technology, National NanoFab Center, 291 Daehak-ro, Yuseong-gu, Daejeon 34141, Republic of Korea

4 Department of Nano-Mechatronics, University of Science and Technology (UST), 217 Gajeong-ro, Yuseong-gu, Daejeon 34113, Republic of Korea

5 Bionanotechnology Research Center, KRIBB, 125 Gwahak-ro, Yuseong-Gu, Daejeon 34141, Republic of Korea

6 Department of Nanobiotechnology, KRIBB School of Biotechnology, UST, 217 Gajeong-ro, Yuseong-gu, Daejeon 34113, Republic of Korea

The flu is one of the most common infectious diseases caused by influenza viruses [1,2]. As influenza viruses circulate year-round with high infectivity, rapid and precise diagnosis of influenza viruses is important to prevent the spreading of infectious diseases [3-5]. For the detection of influenza viruses, traditional cell culture and polymerase chain reaction methods have been employed [6,7]. Although these approaches are precise and powerful, they are unsuitable for rapid point-of-care diagnosis because they are timeconsuming, require complicated equipment, and involve labor-intensive processes [6, 7]. Rapid immunoassays have also been widely used for the quick diagnosis of influenza virus-infected patients [8-10]. More recently, several advanced mechanical, electrical, and optical immunosensing approaches have been developed and have contributed to the sensitive, selective, and label-free detection of infectious factors [11-13]. Among these approaches, simple optical biosensors based on nanostructures have attracted attention because of their rapidity and sensitivity and because they do not require direct contact [14-17].

Nanopillar structures composed of a periodic arrangement of regularly shaped materials on a substrate have attracted increasing attention as optical sensing materials because they can interact with light via diffractive grating 
or reflection [18-21]. In addition, nanopillar structures can be fabricated by nanoimprint lithography, a two-dimensional nanostructure manufacturing technique that enables simple, low-cost, and high-throughput production [22-26]. Based on the excellent properties of nanopillar structures, Charlton et al. developed optical field-enhanced silicon nanopillars for the detection of DNA [27]. Lee et al. reported a localized surface plasmon resonance nanopillar biosensor prepared by nanoimprint lithography [28]. An SU-8 nanopillar array-based optical biosensor was also developed for the label-free detection of bovine serum albumin [18]. Various nanopillar-based optical biosensors have been developed, but a nanopillar-based biosensor for infectious viruses has not yet been developed.

Herein, we present a nanopillar-based optical biosensor for the detection of influenza A viruses via biomimetic surface functionalization of polymerized dopamine (PDA) and sialic acid-mimic peptide (SA-peptide). PDA, a well-known universal biomimetic adhesive, was coated onto the surfaces of the nanopillar structures [29-31]. The SA-peptide, a receptor developed against the hemagglutinin (HA) of influenza viruses from phage-displayed random peptide libraries, was conjugated to the PDA-coated nanopillar structures [32-36]. We characterized the process of the biomimetic surface functionalization of nanopillar structures by X-ray photoelectron spectroscopy (XPS) and contact angle measurements. Importantly, we found that the reflectance of the nanopillar structure was correlated with the binding of influenza viruses. Consequently, the SA-peptide-functionalized nanopillar structure can be used for the detection of influenza A viruses through the simple measurement of reflectance. The reflectance of the nanopillar biosensor changed with increasing concentration of the virus, enabling the detection of influenza viruses in the range of $10^{3}-10^{5}$ plaque-forming units (PFUs). Moreover, it was verified that the developed nanopillar biosensor uniquely identified influenza A viruses. We anticipate that the biomimetic nanopillar-based optical biosensor can be a simple alternative method for influenza virus detection.

\section{Materials and Methods}

\subsection{Materials}

Dopamine chloride $\left(\mathrm{C}_{9} \mathrm{H}_{11} \mathrm{NO}_{2} \cdot \mathrm{HCl}\right)$, Tris- $\mathrm{HCl}$ buffer (pH 8.5), dimethyl sulfoxide $\left(\mathrm{C}_{2} \mathrm{H}_{6} \mathrm{OS}\right)$, and ethanolamine $\left(\mathrm{C}_{2} \mathrm{H}_{7} \mathrm{NO}\right)$ were purchased from Sigma-Aldrich (USA). SApeptide (Fmoc-ARLSPTMVHPNGAQP-NH ${ }_{2}$ ) and control peptide (Fmoc-CDDYYYGFGCNKFCRPR-NH $\mathrm{N}_{2}$ ) were purchased from PEPTRON Corp. (Korea). Influenza virus type A H1N1 (A/California/07/2009, pandemic H1N1), H3N2 (A/Brisbane/10/2007, seasonal H3N2), H5N2 (A/ aquatic birds/Korea/W351/2008, avian H5N2), and influenza virus type B (IFVB) (B/Victoria/Brisbane/60/2008) were provided by the Bio Nano Health Guard Research Center (H-GUARD) of Korea. The viral titer of the stock was determined in Madin-Darby canine kidney cells via a plaque assay.

\subsection{Fabrication of Nanopillar Structures}

Nanopillar structures were fabricated by nanoimprint lithography (Fig. 1). In detail, a 4-inch silicon wafer was cleaned with a plasma asher for $10 \mathrm{~min}$ and spin-coated with IC1-500 spin-on-glass (SOG) (Futurrex, Inc., USA) at $3000 \mathrm{rpm}$ for $30 \mathrm{~s}$. The hole-patterned silicon stamp (diameter of $150 \mathrm{~nm}$, depth of $300 \mathrm{~nm}$, and gap width of $450 \mathrm{~nm}$ ) was pressed on the SOG-coated wafer at $2000 \mathrm{kgf} \mathrm{cm}^{-2}$ at $150{ }^{\circ} \mathrm{C}$ for $10 \mathrm{~min}$ using an ANT-6HO2 UV/thermal nanoimprint lithography system (KIMM, Korea). Next, the whole stamp was detached from the wafer to obtain nanopillar structures on the wafer.

\subsection{Surface Functionalization of Nanopillar Structures}

The nanopillar structures were immersed in a dopamine solution $\left(2.0 \mathrm{mg} \mathrm{mL}^{-1}\right.$ in $1 \times 10^{-3} \mathrm{M}$ Tris- $\mathrm{HCl}$ buffer, $\mathrm{pH}$ 8.5 ) for $2 \mathrm{~h}$ at $25^{\circ} \mathrm{C}$, washed with deionized (DI) water, and dried with argon gas. Next, SA-peptide or control peptide $\left(1.0 \mathrm{mg} \mathrm{mL}^{-1}\right.$ in DI water, $\left.50 \mu \mathrm{L}\right)$ was dropped onto the PDA-coated nanopillar structures at $25{ }^{\circ} \mathrm{C}$. After $2 \mathrm{~h}$, the structures were washed with DI water and dried with argon gas. The peptide-functionalized nanopillar structures were blocked with ethanolamine $\left(1 \times 10^{-3} \mathrm{M}, 200 \mu \mathrm{L}, \mathrm{pH} 9\right)$, washed with DI water, and dried with argon gas.

\subsection{Detection of Influenza Viruses Using Nanopillar}

To detect influenza viruses, the peptide-functionalized nanopillar structures were incubated with $20 \mu \mathrm{L}$ of a viral solution for $1 \mathrm{~h}$ at $37^{\circ} \mathrm{C}$. After the nanopillar structures were washed with DI water, the reflectance was measured.

\subsection{Instrumentation}

Scanning electron microscopy (SEM) images were obtained with Quanta 250 FEG (FEI, USA) at an acceleration voltage of $10 \mathrm{kV}$. Reflectance was measured using a spectrometer (FLAME-S; Ocean Optics, USA). Contact angle was measured with Phoenix 300 Plus (SEO Co., Ltd, Korea). XPS was obtained using PHI 5000 Versa Probe (Ulvac-PHI, Japan). 


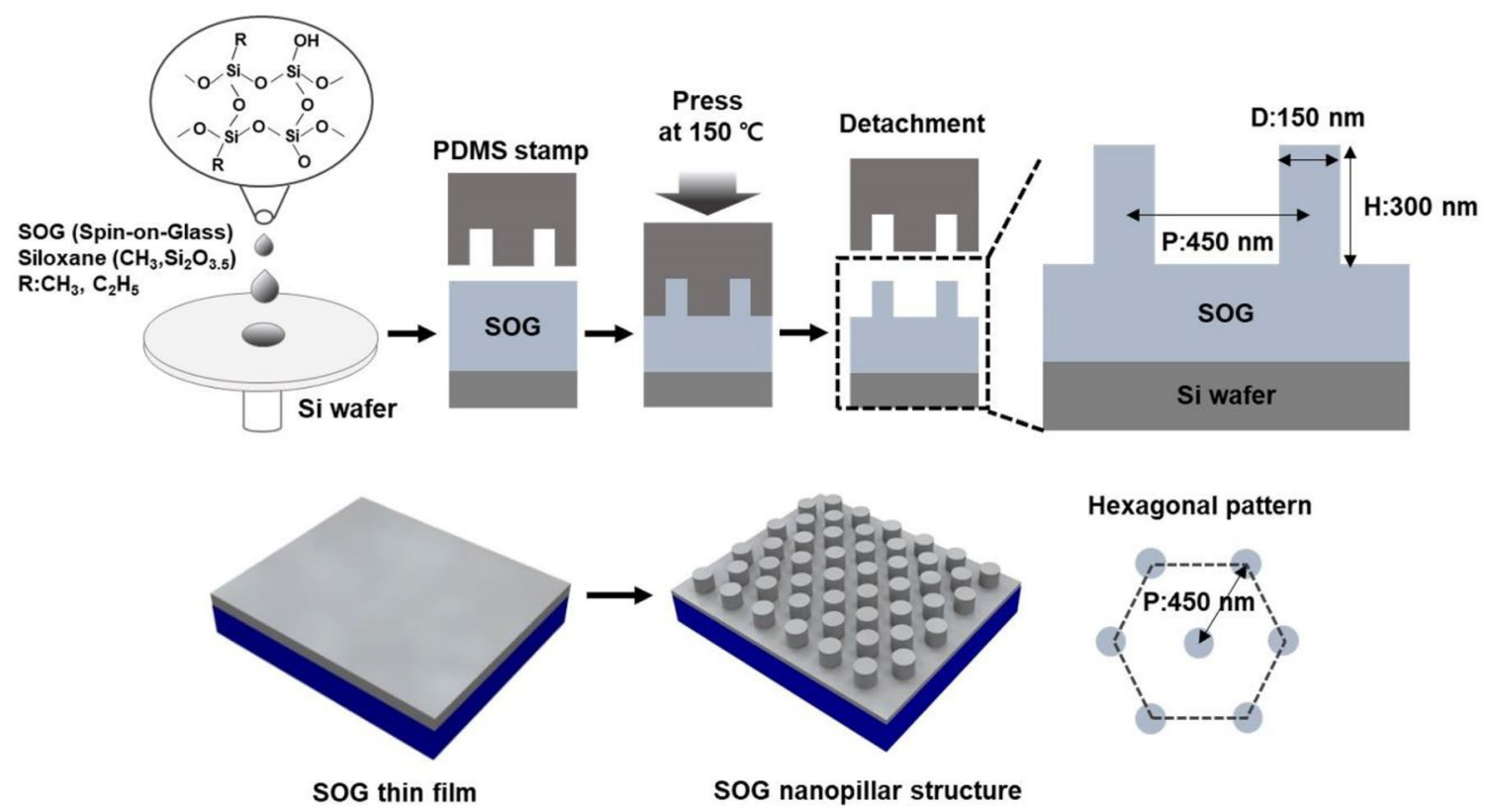

Fig. 1 Schematic illustration of the fabrication procedure of SOG nanopillar structures

\section{Results and Discussion}

The nanopillar structures used in this work were fabricated by nanoimprint lithography. Briefly, the SOG sol was deposited on the Si substrate by a spinning technique. A nanostructure template was formed by the sol-gel transition of the SOG. After the imprinting of a hole-patterned stamp, pillar-shaped nanostructures were obtained on the substrate. To characterize the fabricated nanopillar structures, we observed their morphology by SEM. As shown in Fig. 2a and b, the SOG was uniformly patterned on the Si substrate with a diameter of $150 \mathrm{~nm}$, a height of $300 \mathrm{~nm}$, and a gap width of $450 \mathrm{~nm}$. Note that
Fig. 2 SEM images of nanopillar structures from the a top view and $\mathbf{b}$ side view. $\mathbf{c}$ Reflectance spectrum of nanopillar structures. d Reflectance shift as a function of PDA polymerization time. Data represent average plus standard deviation from three measurements a
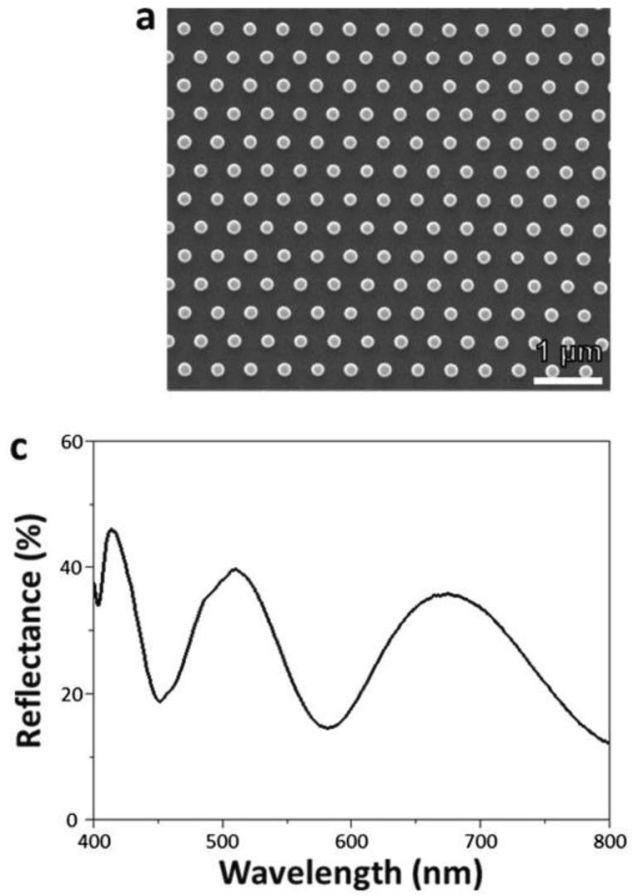
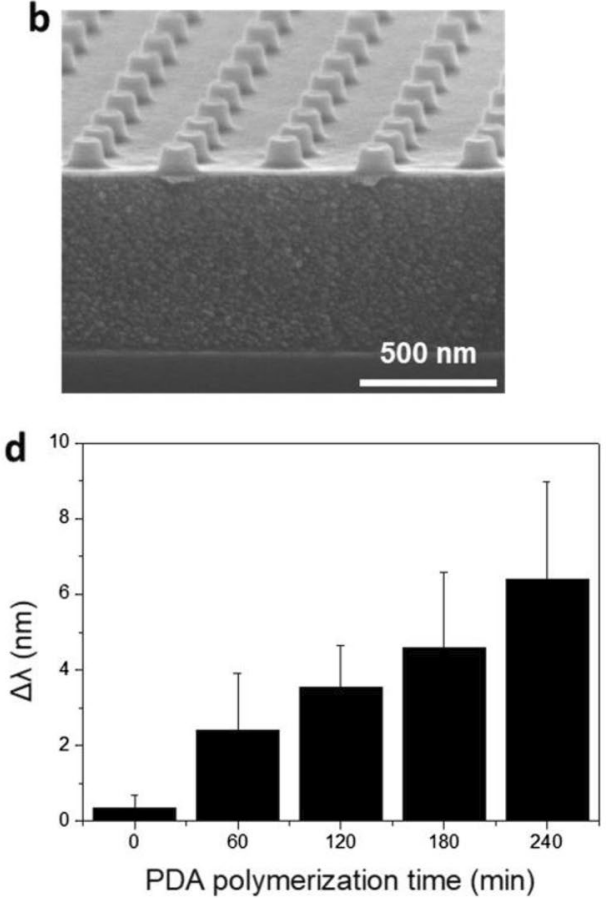
the nanopillar structures were constructed on a wafer scale with high reproducibility. Previously, it was reported that the nanopillar structures have interesting photonic crystallike properties in terms of light reflectance and diffraction, which can be altered by surface modification and interaction with molecules $[18,37]$. The constructed nanopillar structures also exhibited distinct peaks in the visible region (Fig. 2c). As these peaks can be determined by the refractive index near the nanopillar structures, we adopted the nanopillar structures as a label-free optical biosensing platform. Figure $2 \mathrm{~d}$ is the reflectance shift of nanopillar structures depending on PDA polymerization time. The refractive index increased as increasing the thickness of PDA [29, 38], resulting in the reflectance shift of the nanopillar structure. This suggests that the nanopillar structure can be utilized in the detection of the virus.

For the construction of the nanopillar-based optical biosensor, the surfaces of the nanopillar structures were biomimetically modified by PDA and SA-peptide sequentially (Fig. 3a). First, the nanopillar structures were incubated in a dopamine solution and washed. Dopamine, an adhesive molecule secreted by mussels, is a compound of catechol (1,2-dihydroxybenzene) and a primary amine functional group [29, 31]. It is well known that PDA-coated surfaces can be formed by simply immersing substrates in an aqueous solution of dopamine at $\mathrm{pH} 8.5$ [30, 39-42]. Next, SA-peptide was immobilized onto the PDA-coated nanopillar structures via Michael addition or Schiff base reaction between the N-terminus of the peptide and PDA [43]. In general, influenza viral infection is initiated by the binding of HA to cell surface receptors containing sialic acid. Therefore, the SA-peptide can be a bioreceptor for influenza viruses through hydrogen bonding or hydrophobic interactions between HA and sialic acid [32]. Following SA-peptide immobilization, the nanopillar structures were blocked by ethanolamine to prevent nonspecific binding. This surface functionalization process is a simple and rapid way of immobilizing bioreceptors on a nanostructured surface without chemical linkers, suggesting the potential to interface several types of biomolecules with nanostructures routinely.

To verify the surface functionalization of the nanopillar structures, contact angle measurements, reflectance spectrophotometry, and XPS analyses were performed. Figure $3 \mathrm{~b}$ shows the water contact angle measurements for bare, PDA-coated, SA-peptide-immobilized, and ethanolamineblocked nanopillar structures. The wettability of the nanopillar structures changed from hydrophobic to hydrophilic after PDA coating because of the dihydroxyl groups of PDA. The average contact angle was $112.85 \pm 4.03^{\circ}$ on the bare nanopillar structures and $41.52 \pm 0.87^{\circ}$ on the PDA-coated nanopillar structures. After SA-peptide immobilization and ethanolamine blocking, the contact angles were further decreased to $40.51 \pm 0.91^{\circ}$ and $36.44 \pm 2.42^{\circ}$, respectively. This indicated that PDA was successfully deposited onto the nanopillar structures and formed stable conjugation with the SA-peptide. The reflectance spectra of bare, PDA-coated, SA-peptide-immobilized, and ethanolamine-blocked nanopillar structures were also obtained (Fig. 3c). Compared with the bare nanopillar structures, the reflectance spectrum of the PDA-coated nanopillar was red-shifted to $7.00 \pm 1.4 \mathrm{~nm}$. However, the reflectance spectra of the SA-peptide-immobilized and ethanolamine-blocked nanopillar structures were barely changed $(0.65 \pm 1.80 \mathrm{~nm}$ for SA-peptide and $-0.77 \pm 2.37 \mathrm{~nm}$ for ethanolamine) compared with the PDAcoated nanopillar structures. This is attributed to the PDA deposition on the nanopillar structures, which increased
Fig. 3 a Schematic illustration of biomimetic surface functionalization procedure of nanopillar structures via PDA and SA-peptide. b Water contact angle and $\mathbf{c}$ reflectance spectra of bare, PDA-coated, SA-peptide-immobilized, and ethanolamine-blocked nanopillar structures. Water contact angle data represent average plus standard deviation from three measurements a
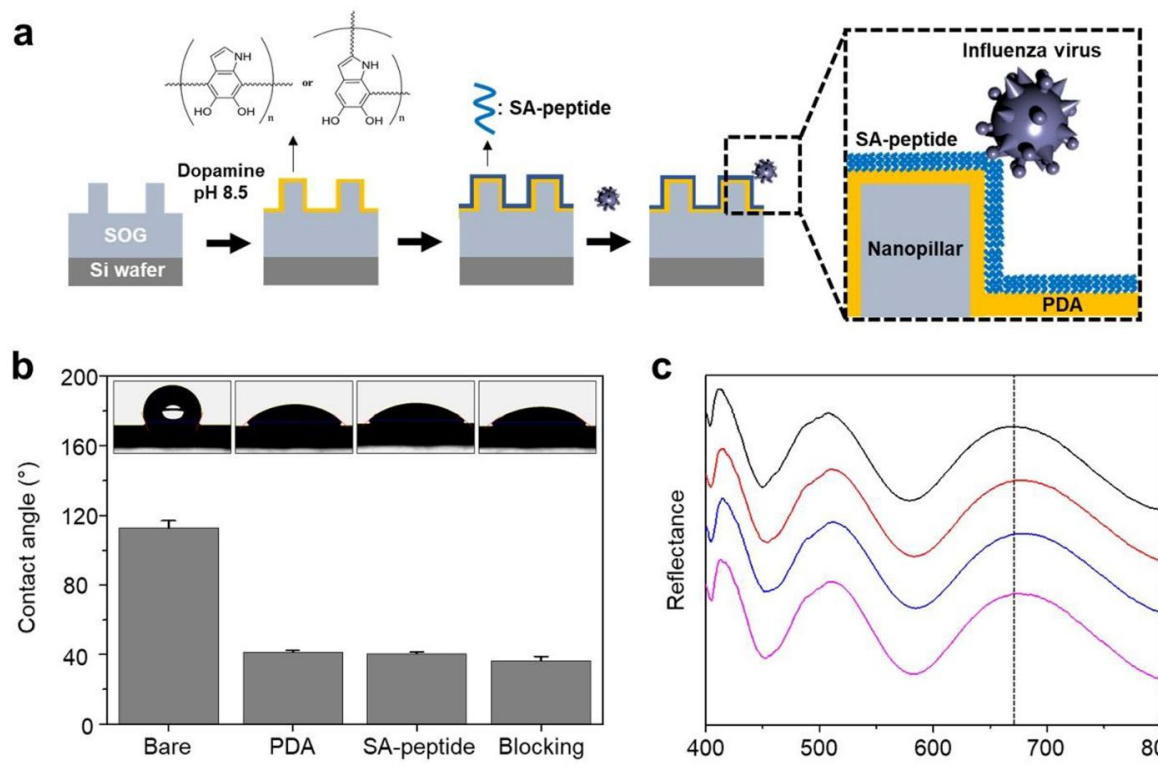

C

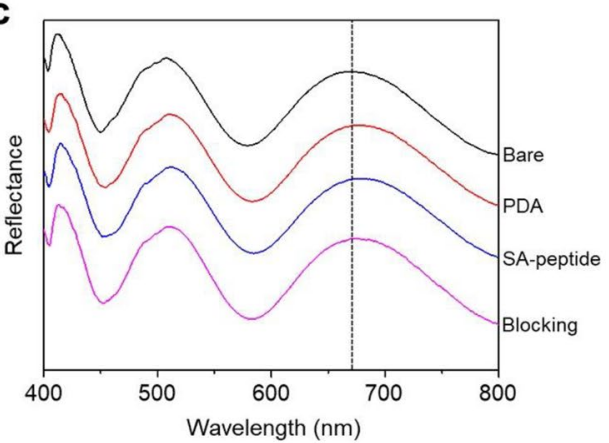


the refractive index from 1.42-1.43 (SOG) to 1.55 (PDA). Moreover, small molecules such as SA-peptide and ethanolamine were less effective in altering the refractive index of the nanopillar structures.

To analyze the atomic composition of the nanopillar structures, XPS was carried out. As shown in Table 1, the nitrogen content increased from $0.46 \%$ to $6.99 \%$ after PDA coating. The nitrogen and oxygen content further increased to $7.57 \%$ and $26.62 \%$, respectively, after SA-peptide immobilization. The composition of carbon increased to $67.00 \%$ after the blocking process because of the primary alcohol. The XPS results indicated that the nanopillar structures were successfully functionalized by PDA, SA-peptide, and ethanolamine. PDA is used not only for the surface modification of nanopillar structures but also for the immobilization of the SA-peptide by acting as a double-sided tape. In our experiment, biomimetic nanopillar structures were fabricated by PDA coating and SA-peptide immobilization, allowing us to detect influenza A viruses.

After surface functionalization of the nanopillar structures, we performed label-free detection of influenza viruses. As the size of the influenza virus is $80-120 \mathrm{~nm}$, the reflectance of the nanopillar biosensor could be altered by capturing the virus. Figure $4 \mathrm{a}$ shows the shift in reflectance versus the peptide type. When the SA-peptide was immobilized onto the nanopillar structures, the reflectance of the nanopillars was shifted to $6.38 \pm 0.53$ upon detection of the influenza virus (10 [5] PFU). The inset shows that the virus can be captured on the nanopillar biosensor. When the control peptide consisting of random sequences was attached to the nanopillars, the reflectance was shifted by $0.98 \pm 0.65 \mathrm{~nm}$ upon the detection of the same virus. This clearly showed that influenza viruses could be detected by the SA-peptideimmobilized nanopillar structures.

The SA-peptide selectively recognizes HA on the membranes of influenza type A viruses, and thus various influenza type A viruses can be detected by the SApeptide-immobilized nanopillar optical biosensor [32]. It is important to accurately identify influenza A viruses because they are the only viruses known to cause flu pandemics $[1,44]^{\cdot}$ To examine the selectivity of the nanopillar biosensors, they were incubated with four types of viruses

Table 1 Surface atomic composition of bare, PDA-coated, SA-peptide-immobilized, and ethanolamine-blocked nanopillar structures

\begin{tabular}{llll}
\hline & $\mathrm{C}(\%)$ & $\mathrm{O}(\%)$ & $\mathrm{N}(\%)$ \\
\hline Nanopillar & 45.00 & 54.54 & 0.46 \\
Nanopillar/PDA & 69.12 & 23.89 & 6.99 \\
Nanopillar/PDA/SA-peptide & 65.81 & 26.62 & 7.57 \\
$\begin{array}{l}\text { Nanopillar/PDA/SA-peptide/etha- } \\
\text { nolamine }\end{array}$ & 67.00 & 26.60 & 6.40 \\
\hline
\end{tabular}

(H1N1, H3N2, H5N2, and IFVB, $10^{4}$ PFU) and the change in reflectance was observed (Fig. 4b). The reflectance of the nanopillar biosensors was shifted only in the presence of influenza type A viruses $(5.17 \pm 1.49 \mathrm{~nm}, 4.51 \pm 1.06 \mathrm{~nm}$, and $6.36 \pm 0.68 \mathrm{~nm}$ for $\mathrm{H} 1 \mathrm{~N} 1, \mathrm{H} 3 \mathrm{~N} 2$, and $\mathrm{H} 5 \mathrm{~N} 2$, respectively). In the presence of IFVB, the reflectance shifted slightly. These observations indicate that the fabricated nanopillar biosensors can accurately recognize influenza type A viruses.

The viral dose-dependent change in the reflectance of the nanopillar biosensors is shown in Fig. 4c, wherein the reflectance peaks were shifted depending on the concentration of the influenza virus. Figure $4 d$ shows the reflectance shift as a function of viral concentration, illustrating that the reflectance variation increased from $1.10 \pm 0.19 \mathrm{~nm}$ to $7.03 \pm 1.70 \mathrm{~nm}$ as the viral concentration increased from $10^{3}$ to $10^{5} \mathrm{PFU}$. This result demonstrates that the fabricated nanopillar biosensor can realize the label-free detection of influenza type A viruses with a detection limit of $10^{3} \mathrm{PFU}$. The sensitivity of the nanopillar biosensor is comparable with that of photonic crystal-based label-free biosensors for HIV, Ebola, and Vaccinia viruses [12, 16]. Previously, several kinds of methods have been developed for the identification of influenza viruses including molecular diagnostics and immunoassays [45]. They suggested the sensitive detection of viruses at a lower concentration than $100 \mathrm{PFU}$ [45]. Although the current method showed the detection limit of $10^{3} \mathrm{PFU}$, it has the advantages of simple fabrication and detection procedures. Also, the nanopillar biosensor provided a higher sensitivity compared with the electrochemical sensor with SA-peptide [46].

We fabricated a label-free optical biosensor based on nanopillar structures and SA-peptide immobilization for the detection of influenza viruses. This sensor has the advantage of being applicable to point-of-care testing because it can detect the whole virus without pre-treatment [46]. In particular, influenza A viruses can cause devastating public outbreaks because of the high infectiousness between species $[47,48]$. Therefore, early detection is required to prevent the spreading of influenza A viruses. Although influenza A viruses possess unpredictability and transmutability, HA is commonly found on these viruses. Consequently, the developed sensing platform can be used to effectively detect influenza A viruses.

\section{Conclusion}

In summary, we developed a simple method for the detection of influenza viruses using nanopillar structures, PDA, and SA-peptides. Nanopillar structures were routinely fabricated by nanoimprint lithography, providing a large surface area and photonic crystal-like properties. PDA plays a crucial 
a

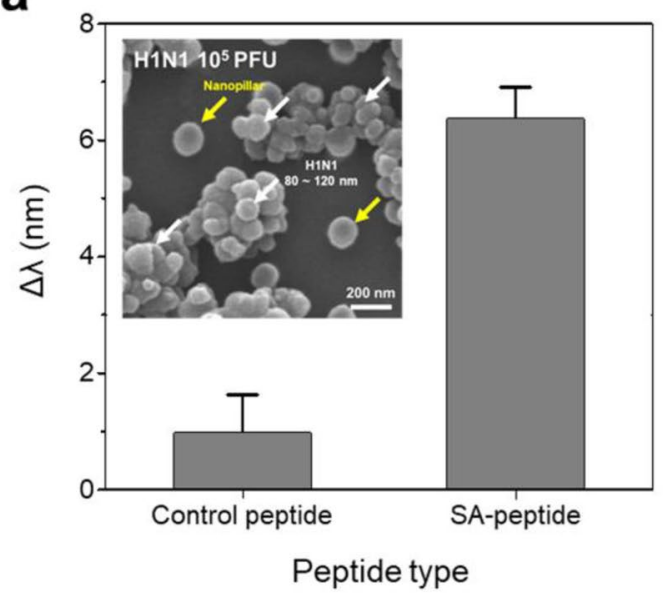

C

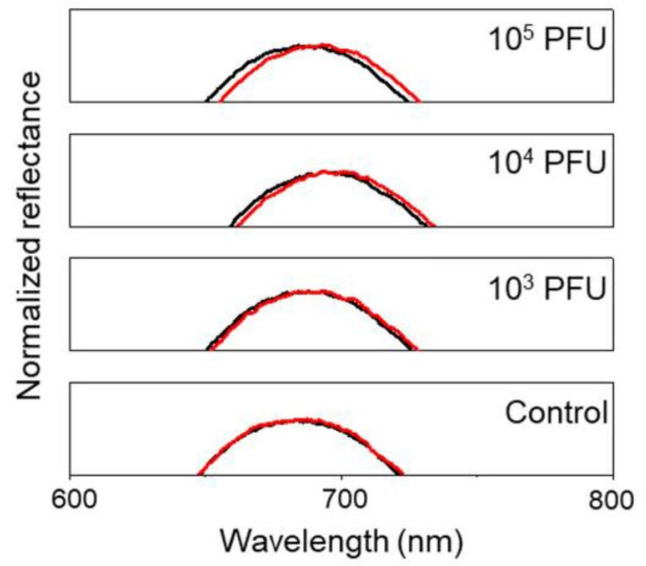

Fig. 4 a Result of influenza virus detection using nanopillar structures immobilized with SA-peptide and control peptide. Inset is an SEM image of nanopillar biosensor after the detection of H1N1 $\left(10^{5} \mathrm{PFU}\right)$. b Selective detection of influenza type A viruses (H1N1, $\mathrm{H} 3 \mathrm{~N} 2$, and H5N2) using nanopillar biosensors. c Reflectance spectra

role in the connection between the nanopillar structures and the SA-peptide, enabling us to successfully fabricate the biosensors. As the HA on influenza viruses can be recognized by the SA-peptide, influenza type A viruses can be detected at a low concentration of $10^{3} \mathrm{PFU}$ using the nanopillar biosensors. It is anticipated that nanopillar biosensors will be useful for the on-site label-free detection of influenza viruses.

Acknowledgements This research was supported by National R\&D Programs through National Research Foundation (NRF) of Korea funded by Ministry of Science and ICT (MSIT) of Korea (NRF-2019R1C1C1006867, NRF-2019R1C1C1006084, NRF2018M3A9E2022821, and NRF-2021M3H4A1A02051048), Global Frontier Program through Center for BioNano Health-Guard funded by MSIT of Korea (H-GUARD_2013M3A6B2078950 and H-GUARD_2013M3A6B2078), Technology Development Program b

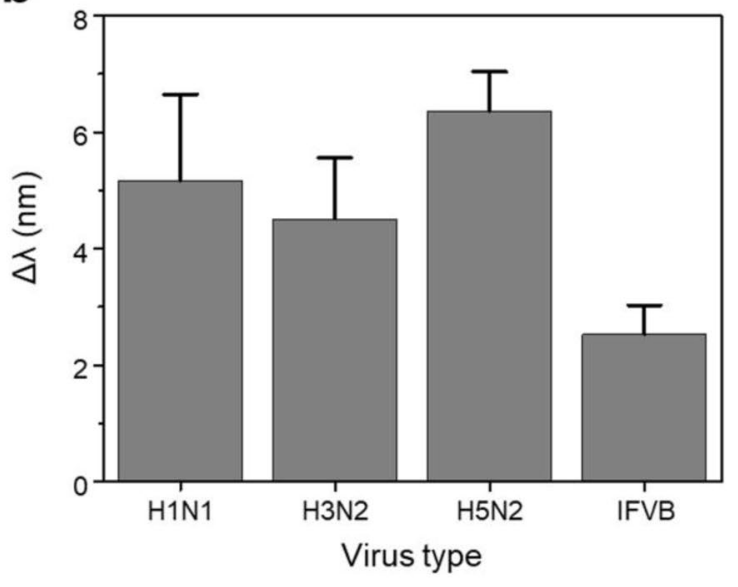

d

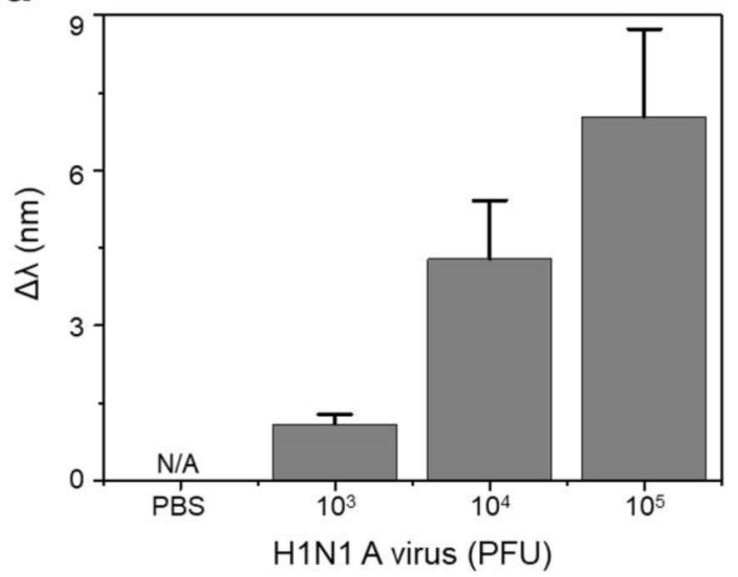

of nanopillar biosensors before (black) and after (red) the detection of influenza viruses. d Reflectance shift as a function of viral concentration. Data represent average plus standard deviation from 10 measurements

for Biological Hazards Management in Indoor Air through Korea Environment Industry \& Technology Institute (KEITI) funded by Ministry of Environment (ME) of Korea (2021003370003), Nanomedical Devices Development Program of National Nano Fab Center, and KRIBB Research Initiative Program (1711134081 and 1711134066).

\section{References}

1. Neumann, G., Noda, T., Kawaoka, Y.: Emergence and pandemic potential of swine-origin H1N1 influenza virus. Nature 459, 931939 (2009)

2. Kilbourne, E.D.: Influenza pandemics of the 20th century. Emerg Infect Dis 12, 9-14 (2006)

3. Yi, S.Y., Lee, U., Chung, B.H., Jung, J.: A scanometric antibody probe for facile and sensitive immunoassays. Chem Commun 51, 8865-8867 (2015) 
4. Vavricka, C.J., Liu, Y., Kiyota, H., Sriwilaijaroen, N., Qi, J., Tanaka, K., Wu, Y., Li, Q., Li, Y., Yan, J., Suzuki, Y., Gao, G.F.: Influenza neuraminidase operates via a nucleophilic mechanism and can be targeted by covalent inhibitors. Nat Commun 4, 1468-1469 (2013)

5. Zhang, X., Dhawane, A.N., Sweeney, J., He, Y., Vasireddi, M., Iyer, S.S.: Electrochemical assay to detect influenza viruses and measure drug susceptibility. Angew Chemie Int Ed 54, 5929_ $5932(2015)$

6. Henkel, J.H., Aberle, S.W., Kundi, M., Popow-Kraupp, T.: Improved detection of respiratory syncytial virus in nasal aspirates by seminested RT-PCR. J Med Virol 53, 366-371 (1997)

7. Jones, L.J., Upson, R.H., Haugland, R.P., Panchuk-Voloshina, N., Zhou, M., Haugland, R.P.: Quenched BODIPY dye-labeled casein substrates for the assay of protease activity by direct fluorescence measurement. Anal Biochem 251, 144-152 (1997)

8. Yu, S.T., Bui, C.T., Nguyen, A.V., Trinh, T.T., Yeo, S.J.: Clinical evaluation of rapid fluorescent diagnostic immunochromatographic test for influenza A virus (H1N1). Sci Rep 8(1), 1-9 (2018)

9. Hwang, S.G., Ha, K., Guk, K., Lee, D.K., Eom, G., Song, S., Kang, T., Park, H., Jung, J., Lim, E.K.: Rapid and simple detection of tamiflu-resistant influenza virus: development of oseltamivir derivative-based lateral flow biosensor for pointof-care (POC) diagnostics. Sci Rep 8, 1-11 (2018)

10. Bamrungsap, S., Apiwat, C., Chantima, W., Dharakul, T., Wiriyachaiporn, N.: Rapid and sensitive lateral flow immunoassay for influenza antigen using fluorescently-doped silica nanoparticles. Microchim Acta 181, 223-230 (2014)

11. Inci, F., Tokel, O., Wang, S., Gurkan, U.A., Tasoglu, S., Kuritzkes, D.R., Demirci, U.: Nanoplasmonic quantitative detection of intact viruses from unprocessed whole blood. ACS Nano 7, 4733-4745 (2013)

12. Yanik, A.A., Huang, M., Kamohara, O., Artar, A., Geisbert, T.W., Connor, J.H., Altug, H.: An optofluidic nanoplasmonic biosensor for direct detection of live viruses from biological media. Nano Lett 10, 4962-4969 (2010)

13. Hwang, H.J., Ryu, M.Y., Park, C.Y., Ahn, J., Park, H.G., Choi, C., Ha, S.D., Park, T.J., Park, J.P.: High sensitive and selective electrochemical biosensor: label-free detection of human norovirus using affinity peptide as molecular binder. Biosens Bioelectron 87, 164-170 (2017)

14. Lee, J., Lcoz, K., Roberts, A., Ellington, A.D., Savran, C.A.: Diffractometric detection of proteins using microbead-based rolling circle amplification. Anal Chem 82, 197-202 (2010)

15. Fenzl, C., Hirsch, T., Wolfbeis, O.S.: Photonic crystals for chemical sensing and biosensing. Angew Chemie Int Ed 53, 3318-3335 (2014)

16. Shafiee, H., Lidstone, E.A., Jahangir, M., Inci, F., Hanhauser, E., Henrich, T.J., Kuritzkes, D.R., Cunningham, B.T., Demirci, U.: Nanostructured optical photonic crystal biosensor for HIV viral load measurement. Sci Rep 4, 1-7 (2014)

17. Couturier, J.P., Sütterlin, M., Laschewsky, A., Hettrich, C., Wischerhoff, E.: Responsive inverse opal hydrogels for the sensing of macromolecules. Angew Chemie Int Ed 54, 6641-6644 (2015)

18. Holgado, M., Barrios, C.A., Ortega, F.J., Sanza, F.J., Casquel, R., Laguna, M.F., Bañuls, M.J., López-Romero, D., Puchades, R., Maquieira, A.: Label-free biosensing by means of periodic lattices of high aspect ratio SU-8 nano-pillars. Biosens Bioelectron 25, 2553-2558 (2010)

19. Chen, J.K., Zhou, G.Y., Chang, C.J.: Real-time multicolor antigen detection with chemoresponsive diffraction gratings of silicon oxide nanopillar arrays. Sens Actuat B Chem 186, 802-810 (2013)

20. Oh, Y.J., Kim, J.J., Jeong, K.H.: Biologically inspired biophotonic surfaces with self-antireflection. Small 10, 2558-2563 (2014)
21. Purtov, J., Rogin, P., Verch, A., Johansen, V.E., Hensel, R.: Nanopillar diffraction gratings by two-photon lithography. Nanomaterials 9, 1-11 (2019)

22. Kuo, C.W., Shiu, J.Y., Cho, Y.H., Chen, P.: Fabrication of largearea periodic nanopillar arrays for nanoimprint lithography using polymer colloid masks. Adv Mater 15, 1065-1068 (2003)

23. Chou, S., Krauss, P.R., Renstrom, P.J.: Nanoimprint lithography. J Vac Sci Technol B Microelectron Nanom Struct Process Meas Phenom 14, 4129-4133 (1996)

24. Chou, S.Y., Krauss, P.R., Renstrom, P.J.: Imprint lithography with 25-nanometer resolution. Science 272, 85-87 (1996)

25. Hirai, Y., Tanaka, Y.: Application of nano-imprint lithography. J Photopolym Sci Technol 15, 475-480 (2002)

26. Lee, J., Park, H.H., Choi, K.B., Kim, G., Lim, H.: Fabrication of hybrid structures using UV roll-typed liquid transfer imprint lithography for large areas. Microelectron Eng 127, 72-76 (2014)

27. Charlton, J.J., Jones, N.C., Wallace, R.A., Smithwick, R.W., Bradshaw, J.A., Kravchenko, I.I., Lavrik, N.V., Sepaniak, M.J.: Nanopillar based enhanced-fluorescence detection of surfaceimmobilized beryllium. Anal Chem 87, 6814-6821 (2015)

28. Lee, S.W., Lee, K.S., Ahn, J., Lee, J.J., Kim, M.G., Shin, Y.B.: Highly sensitive biosensing using arrays of plasmonic Au nanodisks realized by nanoimprint lithography. ACS Nano 5, 897-904 (2011)

29. Lee, H., Dellatore, S.M., Miller, W.M., Messersmith, P.B.: Mussel-inspired surface chemistry for multifunctional coatings. Science 318, 426-430 (2007)

30. Chen, J., Li, Y., Huang, K., Wang, P., He, L., Carter, K.R., Nugen, S.R.: Nanoimprinted patterned pillar substrates for surfaceenhanced raman scattering applications. ACS Appl Mater Interfaces 7, 22106-22113 (2015)

31. Lee, H., Lee, B.P., Messersmith, P.B.: A reversible wet/dry adhesive inspired by mussels and geckos. Nature 448, 338-341 (2007)

32. Matsubara, T., Onishi, A., Saito, T., Shimada, A., Inoue, H., Taki, T., Nagata, K., Okahata, Y., Sato, T.: Sialic acid-mimic peptides as hemagglutinin inhibitors for anti-influenza therapy. J Med Chem 53, 4441-4449 (2010)

33. Sauter, N.K., Wurzburg, B.A., Hanson, J.E., Wiley, D.C., Bednarski, M.D., Whitesides, G.M., Skehel, J.J.: Hemagglutinins from two influenza virus variants bind to sialic acid derivatives with millimolar dissociation constants: A 500-MHz proton nuclear magnetic resonance study. Biochemistry 28, 8388-8396 (1989)

34. Matsubara, T., Ujie, M., Yamamoto, T., Akahori, M., Einaga, Y., Sato, T.: Highly sensitive detection of influenza virus by borondoped diamond electrode terminated with sialic acid-mimic peptide. Proc Natl Acad Sci USA 113, 8981-8984 (2016)

35. Lim, E.K., Guk, K., Kim, H., Chung, B.H., Jung, J.: Simple, rapid detection of influenza A (H1N1) viruses using a highly sensitive peptide-based molecular beacon. Chem Commun 52, 175-178 (2016)

36. Song, S., Ha, K., Guk, K., Hwang, S.G., Choi, J.M., Kang, T., Bae, P., Jung, J., Lim, E.K.: Colorimetric detection of influenza A (H1N1) virus by a peptide-functionalized polydiacetylene (PEPPDA) nanosensor. RSC Adv 6, 48566-48570 (2016)

37. Li, W., Wang, S., He, S., Wnag, J., Guo, Y., Guo, Y.: Enhanced photoluminescence from $\mathrm{CdS}$ with $\mathrm{SiO}_{2}$ nanopillar arrays. Sci Rep 5, $11375(2015)$

38. Vega, M., Martín del Valle, E.M., Pérez, M., Pecharromán, C., Marcelo, G.: Color engineering of silicon nitride surfaces to characterize the polydopamine refractive index. ChemPhysChem $\mathbf{1 9}$, 3418-3424 (2018)

39. Waite, J.H.: Surface chemistry: mussel power. Nat Mater 7, 8-9 (2008)

40. Wang, Z., Chen, L., Wang, Y., Chen, X., Zhang, P.: Improved cell adhesion and osteogenesis of op-HA/PLGA COMPOSITE by poly(dopamine)-assisted immobilization of collagen mimetic 
peptide and osteogenic growth peptide. ACS Appl Mater Interfaces 8, 26559-26569 (2016)

41. Toma, M., Tawa, K.: Polydopamine thin films as protein linker layer for sensitive detection of interleukin- 6 by surface plasmon enhanced fluorescence spectroscopy. ACS Appl Mater Interfaces 8, 22032-22038 (2016)

42. Wang, C., Zhou, J., Wang, P., He, W., Duan, H.: Robust nanoparticle-DNA conjugates based on mussel-inspired polydopamine coating for cell imaging and tailored self-assembly. Bioconjug Chem 27, 815-823 (2016)

43. Ding, Y.H., Floren, M., Tan, W.: Mussel-inspired polydopamine for bio-surface functionalization. Biosurf Biotribol 2, 121-136 (2016)

44. Khatri, M., Dwivedi, V., Krakowka, S., Manickam, C., Ali, A., Wang, L., Qin, Z., Renukaradhya, G.J., Lee, C.-W.: Swine influenza $\mathrm{H} 1 \mathrm{~N} 1$ virus induces acute inflammatory immune responses in pig lungs: a potential animal model for human H1N1 influenza virus. J Virol 84, 11210-11218 (2010)
45. Dalal, A., Mohan, H., Prasad, M., Pundir, C.S.: Detection methods for influenza A H1N1 virus with special reference to biosensors: a review. Biosci Rep 40, 1-18 (2020)

46. Nidzworski, D., Pranszke, P., Grudniewska, M., Król, E., Gromadzka, B.: Universal biosensor for detection of influenza virus. Biosens Bioelectron 59, 239-242 (2014)

47. Nobusawa, E., Sato, K.: Comparison of the mutation rates of human influenza A and B viruses. J Virol 80, 3675-3678 (2006)

48. Zambon, M.C.: Epidemiology and pathogenesis of influenza. J Antimicrob Chemother 44, 3-9 (1999)

Publisher's Note Springer Nature remains neutral with regard to jurisdictional claims in published maps and institutional affiliations. 